\title{
Untersuchungen über den Mechanismus der Magenentleerung bei Mensch und Ratte nach oraler Applikation verschiedener Zucker
}

\author{
Heltmut Mehnert and Harald Förster \\ Medizinische Poliklinik der Universität München \\ (Direktor: Prof. Dr. med. W. SEITz)
}

Eingegangen am 27. Januar 1967

\begin{abstract}
Study of the evacuation mechanism of the stomach after oral administration of different sugars in man and rat
\end{abstract}

Summary. It is the purpose of the present work to describe the relation between the concentration of different test-substancos (glucose, galactose, fructose, saccharose, maltose, lactose, sorbitol and xylitol) and the speed of their evacuation from the stomach. Experiments with 912 rats showed that the osmotic pressure of the administered solution plays an important role in the regulation of the evacuation of the stomach. In accordance with these views, emptying of the stomach is more retarded after giving invert sugar than after saccharose at the same concentration, for the former has twice the osmotic pressure of the latter due to the disaccharide being completely hydrolyzed to glucose and fructose. The question remains open, however, why disaccharides like maltose, saccharose and lactose show a slower evacuation from the stomach than solutions of glucose, galactose or sorbitol at the same concentration, although the latter have twice the osmotic pressure. Viewed separately though, every sugar follows the rule that higher concentrations of the solutions slow down emptying of the stomach. - In a first experiment 19 subjects each received $250 \mathrm{ml}$ of a solution of barium sulphate ; later the subjects were divided into two groups, one receiving the same amount of the contrast medium as before but in addition $50 \mathrm{~g}$ of glucose, the second the contrast and $100 \mathrm{~g}$ of glucose. X-ray pictures of the stomach were taken after $2 \mathrm{~h}$ in each group and the amount of residual filling compared with that of the first experiment. In spite of considerable variation, there was a net slowing of stomach emptying, which was significantly greater after $100 \mathrm{~g}$ of glucose than after $50 \mathrm{~g}$.

Etude du mécanisme d'évacuation de l'estomac chez l'homme et le rat après administration orale de divers sucres

Résumé. Le présent travail se propose de décrire les relations entre la concentration de différentes substances d'essai (glucose, galactose, fructose, saccharose, maltose, lactose, sorbitol, xylitol) et la vitesse d'évacuation de l'estomac. Au cours d'expériences sur 912 rats, on a pu constater que la pression osmotique des solutions administrées jouait un rôle important dans la régulation de l'évacuation de l'estomac. Ainsi, avec le sucre interverti, l'évacuation de l'estomac est retardée par rapport à celle observée avec le saccharose, car le glucose et le fructose se trouvent sous forme libre, et la pression osmotique pour une même concentration des solutions est, de ce fait, doublée. On ne sait pas pourquoi les disaccharides tels que le maltose, le saccharose ot le lactose provoquent une évacuation de l'estomac plus lente que des solutions de même concentration de glucose, de galactose et de sorbi- tol, qui ont une pression osmotique deux fois plus élevé. Chaque sucre considéré séparément suit cependant la règle selon laquelle l'évacuation de l'estomac est retardée quand les solutions ont une concentration plus élevée. - Ein outre, 19 personnes ont reçu, au cours d'une épreuve préliminaire, $250 \mathrm{~cm}^{3}$ d'une solution de sulfate de baryum; au cours de l'épreuve principale, un groupe a reçu $50 \mathrm{~g}$ de glucose, et l'autre groupe $100 \mathrm{~g}$ de glucose, dilués dans la même quantité de bouillie opaque. Chaque fois, on a fait au bout de deux heures des radiographies de l'estomac et on a comparé les contenus restants avec ceux de l'épreuve préliminaire. Malgré une grande dispersion des valeurs isolées, on observait un net retard dans l'évacuation de l'estomac lors de l'administration de glucose; après administration de $100 \mathrm{~g}$ de glucose, ce retard était significativement plus grand qu'après administration de $50 \mathrm{~g}$.

Zusammenfassung. Die vorliegende Arbeit sollte die Beziehungen zwischen der Konzentration verschiedener Prüfsubstanzen (Glucose, Galaktose, Fructose, Saccharose, Maltose, Lactose, Sorbit, Xylit) und der Geschwindigkeit der Magenentleerung aufzeigen. Bei Untersuchungen an 912 Ratten konnte festgestellt werden, daß dem osmotischen. Druck der verabreichten Lösungen eine bedeutsame Rolle bei der Regulierung der Magenentleerung zukommt. So ist die Magenentleerung bei Invertzucker gegenüber der bei Saccharose verzögert, da hier Glucose und Fructose in freier Form vorliegen und der osmotische Druck bei gleicher Konzentration der Lösungen infolgedessen doppelt so hoch ist. Warum die Disaccharide Maltose, Saccharose und Lactose eine langsamere Magenentleerung aufweisen als gleichprozentige Glucose-, Galaktose- und Sorbitlösungen, die einen doppelt so hohen osmotischen Druck haben, muß offenbleiben. Für sich allein betrachtet, folgt jedoch jeder Zucker der Regel, daß bei höherer Konzentration der Lösungen die Magenentleerung verzögert ist. - Außerdem erhielten 19 Probanden im Vorversuch $250 \mathrm{ccm}$ Bariumsulfatlösung, im Hauptversuch bekam eine Gruppe $50 \mathrm{~g}$ Glucose, die andere Gruppe $100 \mathrm{~g}$ Glucose, gelöst in der gleichen Menge Kontrastbrei. Jeweils nach zwei Stunden wurden Röntgenaufnahmen des Magens gemacht und die Restfüllungen mit denen des Vorversuchs verglichen. Trotz starker Streuung der Einzelwerte war bei Zusatz von Glucose eine deutliche Verzögerung der Magenentleerung zu beobachten, die bei Gabe von $100 \mathrm{~g}$ Glucose signifikant größer war als bei Gabe von $50 \mathrm{~g}$ GIucose.

Key-words: Stomach, absorption, glucose, galactose, fructose, saccharose, maltose, lactose, sorbitol, xylitol.
Auf eine Abhängigkeit der Magenentleerung vom osmotischen Druck, also von der Konzentration der verabreichten Lösung, war verschiedentlich hingewie- sen worden $[2,7,13,14,15,16,17,18,20,25,26,27$, 28, 31]. Da besonders im Zusammenhang mit den für Klinik und Praxis wichtigen oralen Glucosebelastun- 
gen diese Frage von Interesse ist, führten wir erneut Untersuchungen bei Mensch und Tier durch. An Ratten wurden Glucose, Galaktose, Fructose, Saccharose, Maltose, Invertzucker, Sorbit und Xylit in verschiedenen Konzentrationen per Schlundsonde verabreicht und die Magenentleerung zu verschiedenen Zeitpunkten untersucht. - Beim Menschen wurde der Einfluß verschiedener Glucosekonzentrationen auf die Magenentleerung geprüft.

\section{Untersuchungsgut und Methodik}

A. Methodik der tierexperimentellen Untersuchungen: Für die tierexperimentellen Untersuchungen wurden 912 männliche Albinoratten herangezogen. Es wurde nach folgender, bereits früher von uns beschriebenen Versuchsanordnung vorgegangen [21, 22]. 24 Std vor Versuchsbeginn wurden zwischen 170 und 240 g schwere Ratten gewogen; sie erhielten von nun an keine Nahrung mehr. Die zu prüfenden Lösungen wurden erst unmittelbar vor Versuchsbeginn hergestellt. Sie enthielten immer $10 \%$ Kolloidon als nicht resorbierbare Kontrollsubstanz sowie die verschiedenen Prüfsubstanzen (Monosaccharide, Disaccharide und Polyole) in verschiedenen Konzentrationen [21, 22]. Zu Versuchsbeginn erhielten die Ratten eine leichte und kurze Äthernarkose. Mit einer Schlundsonde wurden dann $1.3 \mathrm{ml}$ der erwärmten Versuchslösung in den $\mathrm{Ma}$ gen eingebracht. Nach 15, 30 und 60 Min Resorptionsdauer wurden die Tiere durch Ätherinhalation getötet. Nach Eröffnung des Abdomens wurden am Übergang des Dünndarms in das Coecum, am Pylorus und an der Cardia Ligaturen gelegt, der Darm vorsichtig herausgelöst, in Eiswasser abgekühlt und abgewaschen. Anschließend wurden der Mageninhalt und der Darminhalt herausgewaschen. Utber das im Darmlumen wiedergefundene Kollidon wurde das Darmangebot (= Magenentleerung) bestimmt. Der Mageninhalt diente als grobe Kontrolle.

B. Methodik der Untersuchungen am Menschen: Die Versuche wurden an zwei Gruppen von gesunden Personen verschiedenen Alters und Geschlechts durchgeführt. Die nüchternen Probanden bekamen im Kontrollversuch gewöhnlichen Kontrastbrei zu trinken (250 ccm, 2 Volumenteile Bariumsulfat, 1 Volumenteil Wasser). Zwei Stunden danach wurden Röntgenaufnahmen des Magens gemacht, um die zu diesem Zeitpunkt noch bestehende Füllung des Magens zu erfassen. Im sogenannten Hauptversuch erhielt eine erste Gruppe von neun Probanden $50 \mathrm{~g}$ Glucose gelöst in Bariumsulfatlösung (s.o.). Eine zweite Gruppe von zəhn Probanden erhielt $100 \mathrm{~g}$ Glucose zusammen mit dom Kontrastmittel. Nach jeweils zwei Stunden wurden wiederum Röntgenaufnahmen des Magens gemacht, um den Grad der Magenentleerung festzustellen. Es wurden jeweils die Restfüllungen des Magens an Hand der Röntgenaufnahmen planimetrisch ausgewertet.
C. Chemische Nachweise: Das Kollidon wurde nach der modifizierten Methode von SCHUBERT $u$. WAGNER nachgewiesen [21, 32]. Der Nachweis der Glucose erfolgte enzymatisch nach der Vorschrift von HoGETT $u$. NrXoN [12]. Die Disaccharide wurden nach vorangehender saurer Hydrolyse über die dabei freiwerdende Glucose bestimmt [24]. Galaktose wurde über die reduzierende Wirkung dieses Zuckers nach Frank u. KIRBERGER nachgewiesen [11]. Der Nachweis der Polyole erfolgte mit der Perjodatmethode in der Modifikation von WEST u. RAPOPOR'T [29].

\section{Ergebnisse}

Bei der Auswertung der tierexperimentellen Befunde läßt sich für jede Substanz zeigen, daß mit zunehmender Konzentration die Magenentleerung geringer wird. Außerdem ist festzustellen (Tabelle 1, Abb. 1), daß nach 15 min bei 10 bis $20 \%$ igen Lösungen im allgemeinen bereits ein Drittel oder sogar die Hälfte der per Schlundsonde verabreichten Lösung sich im Darm befindet. Zwischen 15 und 30 min nimmt in der Regel die Magenentleerung nur wenig zu; nach $60 \mathrm{~min}$ bei 10 bis $20 \%$ igen Lösungen beträgt sie etwa das Doppelte als nach $15 \mathrm{~min}$. Man kann sagen, daß die Magenentleerung in den ersten 15 min etwa genau so groß ist wie in den folgenden $\mathbf{4 5} \mathrm{min}$. Für alle Substanzen erfolgt bei $10 \%$ igen Lösungen unabhängig von der Resorptionsgeschwindigkeit der Prüfsubstanzen innerhalb der ersten 15 min eine etwa gleich große Magenentleerung. Unterschiede zwischen den einzelnen Substanzen werden erst nach $30 \mathrm{~min}$ und besonders nach $60 \mathrm{~min}$ deutlich.

Aus Abb. 2 kann der Einfluß des osmotischen Drucks auf die Magenentleerung ersehen werden. Invertzucker enthält, wie Saccharose, Glucose und Fructose; da jedoch die beiden Zucker in freier Form vorliegen, ist der osmotische Druck dieser Prüfsubstanzlösung doppelt so hoch wie der der Saccharoselösung. Nach $15 \mathrm{~min}$ Prüfzeit sind die Differenzen im Darmangebot zwischen beiden Lösungen und zwischen Maltose noch gering. Nach 30 und 60 min läßt sich aber eine deutliche Differenz erkennen. Invertzuckerlösung wird nur noch wenig in den Darm entleert, während Saccharose und Maltose schnell aus dem Magen in den Darm freigegeben werden.

In den Tabellen 2 und 3 sind die Ergebnisse der Röntgenkontrastuntersuchungen bei freiwilligen Versuchspersonen wiedergegeben. Der Zusatz von $50 \mathrm{~g}$ Glucose zum Röntgenkontrastmittel bewirkt bereits eine signifikante Hemmung der Entleerung des Kontrastmittels aus dem Magen $(p<0.01)$. Wesentlich ausgerägter ist die Entleerungshemmung jedoch bei Zusatz von $100 \mathrm{~g}$ Glucose zum Kontrastmittel $(p<0.001)$. Die Retention des Kontrastmittels wird durch Zusatz von $50 \mathrm{~g}$ Glucose um 77\% vermehrt, durch Zusatz von $100 \mathrm{~g}$ Glucose jedoch um $370 \%$ (nach 2 Std). Die Magenentleerung in beiden Gruppen verhält sich zwar bereits im Vorversuch zufällig unterschiedlich; statistisch ist 
Tabelle 1. Vergleich der Größe des Darmangebotes verschiedener Zucker nach Verabreichung mit der Schlundsonde (jeweils $1.3 \mathrm{ml}$ Lösung) an Ratten in Abhängigkeit von der Prïfzeit sowie von der Konzentration der verabreichten Lösungen. (Mittelwert \pm S. E.M.) Als Darmangebot wird diejenige Substanzmenge bezeichnet, die in der jeweiligen Zeiteinheit aus dem Magen in den Darm gelangt ist (Angabe in Prozent als Anteil der gesamten verabreichten Menge)

\begin{tabular}{|c|c|c|c|}
\hline & 15 min & $30 \mathrm{~min}$ & $60 \mathrm{~min}$ \\
\hline $\begin{array}{l}\text { Galaktose } \\
10 \% \mathrm{ig}\end{array}$ & $\begin{array}{l}43.2 \mathrm{mg} \pm 5.7 \\
33.2 \% \\
n=11\end{array}$ & $\begin{array}{l}92.1 \mathrm{mg} \pm 10.2 \\
70.9 \% \\
n=10\end{array}$ & $\begin{array}{l}98.4 \mathrm{mg} \pm 6.8 \\
75.6 \% \\
n=10\end{array}$ \\
\hline $20 \%$ ig & $\begin{array}{l}93.6 \mathrm{mg} \text { 土 } 8.9 \\
36.1 \% \\
n=11\end{array}$ & $\begin{array}{l}111.8 \mathrm{mg} \pm 7.1 \\
43.0 \% \\
n=10\end{array}$ & $\begin{array}{l}152.8 \mathrm{mg} \pm 16.0 \\
58.8 \% \\
n=11\end{array}$ \\
\hline $50 \%$ ig & $\begin{array}{l}144.5 \mathrm{mg} \pm 15.1 \\
\mathbf{2 2 . 2} \% \\
n=12\end{array}$ & $\begin{array}{l}175.5 \mathrm{mg} \pm 19.6 \\
\mathbf{2 7 . 0} \% \\
n=11\end{array}$ & $\begin{array}{l}314.5 \mathrm{mg} \pm 29.7 \\
48.4 \% \\
n=11\end{array}$ \\
\hline $\begin{array}{l}\text { Glucose } \\
10 \% \mathrm{ig}\end{array}$ & $\begin{array}{l}51.6 \mathrm{mg} \pm 6.5 \\
39.7 \% \\
n=8\end{array}$ & $\begin{array}{l}71.4 \mathrm{mg} \pm 4.8 \\
54.9 \% \\
n=12\end{array}$ & $\begin{array}{l}117.1 \mathrm{mg} \pm 3.4 \\
90.0 \% \\
n=16\end{array}$ \\
\hline $15 \%$ ig & $\begin{array}{l}75.7 \mathrm{mg} \pm 6.3 \\
38.8 \% \\
n=8\end{array}$ & $\begin{array}{l}110.5 \mathrm{mg} \pm 6.9 \\
53.8 \% \\
n=11\end{array}$ & $\begin{array}{l}156.5 \mathrm{mg} \pm 5.9 \\
80.3 \% \\
n=13\end{array}$ \\
\hline $20 \%$ ig & $\begin{array}{l}78.8 \mathrm{mg} \pm 6.8 \\
30.3 \% \\
n=7\end{array}$ & $\begin{array}{l}140.5 \mathrm{mg} \pm 9.9 \\
54.1 \% \\
n=8\end{array}$ & $\begin{array}{l}184.8 \mathrm{mg} \pm 10.7 \\
71.1 \% \\
n=12 \\
\end{array}$ \\
\hline $50 \%$ ig & $\begin{array}{l}142.8 \mathrm{mg} \pm 18.8 \\
21.9 \% \\
n=6\end{array}$ & $\begin{array}{l}186.7 \mathrm{mg} \pm 10.6 \\
28.7 \% \\
n=12\end{array}$ & $\begin{array}{l}306.8 \mathrm{mg} \pm 15.9 \\
56.6 \% \\
n=12\end{array}$ \\
\hline $\begin{array}{l}\text { Fructose } \\
10 \% \text { ig }\end{array}$ & $\begin{array}{l}54.6 \mathrm{mg} \pm 4.14 \\
42.0 \% \\
n=8\end{array}$ & $\begin{array}{l}70.5 \mathrm{mg} \pm 4.9 \\
54.2 \% \\
n=10\end{array}$ & $\begin{array}{l}97.0 \mathrm{mg} \pm 3.6 \\
74.8 \% \\
n=9\end{array}$ \\
\hline $15 \%$ ig & $\begin{array}{l}77.6 \mathrm{mg} \pm 4.9 \\
39.5 \% \\
n=6\end{array}$ & $\begin{array}{l}89.2 \mathrm{mg} \pm 7.3 \\
45.8 \% \\
n=11\end{array}$ & $\begin{array}{l}134.1 \mathrm{mg} \pm 4.4 \\
71.2 \% \\
n=11\end{array}$ \\
\hline $30 \%$ ig & $\begin{array}{l}89.9 \mathrm{mg} \pm 12.2 \\
22.8 \% \\
n=6\end{array}$ & $\begin{array}{l}125.9 \mathrm{mg} \pm 10.4 \\
32.2 \% \\
n=11\end{array}$ & $\begin{array}{l}202.9 \mathrm{mg} \pm 17.4 \\
51.8 \% \\
n=12\end{array}$ \\
\hline $\begin{array}{l}\text { Lactose } \\
\qquad 10 \% \mathrm{ig}\end{array}$ & $\begin{array}{l}53.1 \mathrm{mg} \pm 4.7 \\
40.8 \% \\
n=11\end{array}$ & $\begin{array}{l}74.9 \mathrm{mg} \pm 6.4 \\
57.6 \% \\
n=12\end{array}$ & $\begin{array}{l}82.9 \mathrm{mg} \pm 3.7 \\
63.8 \% \\
n=13\end{array}$ \\
\hline $\begin{array}{r}\text { Saccharose } \\
10 \% \mathrm{ig}\end{array}$ & $\begin{array}{l}48.4 \mathrm{mg} \pm 5.7 \\
37.2 \% \\
n=10\end{array}$ & $\begin{array}{l}57.6 \mathrm{mg} \pm 3.3 \\
\mathbf{4 4 . 5} \% \\
n=10\end{array}$ & $\begin{array}{l}81.2 \mathrm{mg} \pm 5.4 \\
62.4 \% \\
n=10\end{array}$ \\
\hline $20 \%$ ig & $\begin{array}{l}74.3 \mathrm{mg} \pm 7.7 \\
28.6 \% \\
n=10\end{array}$ & $\begin{array}{l}128.6 \mathrm{mg} \pm 13.7 \\
49.5 \% \\
n=10\end{array}$ & $\begin{array}{l}157.4 \mathrm{mg} \pm 8.8 \\
61.0 \% \\
n=10 \\
\end{array}$ \\
\hline $30 \%$ ig & $\begin{array}{l}101.9 \mathrm{mg} \pm 10.9 \\
26.2 \% \\
n=10\end{array}$ & $\begin{array}{l}126.5 \mathrm{mg} \pm 8.6 \\
32.2 \% \\
n=10\end{array}$ & $\begin{array}{l}208.1 \mathrm{mg} \pm 8.2 \\
53.4 \% \\
n=10 \\
\end{array}$ \\
\hline $50 \%$ ig & $\begin{array}{l}134.1 \mathrm{mg} \pm 13.2 \\
20.3 \% \\
n=10\end{array}$ & $\begin{array}{l}168.6 \mathrm{mg} \pm 10.1 \\
26.0 \% \\
n=10\end{array}$ & $\begin{array}{l}310.4 \mathrm{mg} \pm 27.8 \\
47.8 \% \\
n=10\end{array}$ \\
\hline $\begin{array}{l}\text { Maltose } \\
10 \% \mathrm{ig}\end{array}$ & $\begin{array}{l}53.6 \mathrm{mg} \pm 4.4 \\
41.2 \% \\
n=10\end{array}$ & $\begin{array}{l}77.8 \mathrm{mg} \pm 6.0 \\
59.8 \% \\
n=10\end{array}$ & $\begin{array}{l}90.4 \mathrm{mg} \pm 7.6 \\
69.5 \% \\
n=10\end{array}$ \\
\hline $20 \%$ ig & $\begin{array}{l}59.9 \mathrm{mg} \pm 6.6 \\
23.0 \% \\
n=10\end{array}$ & $\begin{array}{l}113.8 \mathrm{mg} \pm 13.9 \\
\mathbf{4 3 . 8} \% \\
n=10\end{array}$ & $\begin{array}{l}202.4 \mathrm{mg} \pm 24.2 \\
77.8 \% \\
n=10\end{array}$ \\
\hline
\end{tabular}


Tabelle 1 (Fortsetzung)

\begin{tabular}{|c|c|c|c|}
\hline & $15 \mathrm{~min}$ & $30 \mathrm{~min}$ & $60 \mathrm{~min}$ \\
\hline $30 \%$ ig & $\begin{array}{l}95.8 \mathrm{mg} \pm 21.4 \\
24.3 \% \\
n=10\end{array}$ & $\begin{array}{l}107.2 \mathrm{mg} \pm 9.9 \\
27.5 \% \\
n=10\end{array}$ & $\begin{array}{l}224.9 \mathrm{mg} \pm 18.7 \\
57.5 \% \\
n=10\end{array}$ \\
\hline $50 \%$ ig & $\begin{array}{l}101.9 \mathrm{mg} \pm 18.2 \\
15.7 \% \\
n=10\end{array}$ & $\begin{array}{l}149.8 \mathrm{mg} \pm 12.4 \\
23.1 \% \\
n=10\end{array}$ & $\begin{array}{l}290.9 \mathrm{mg} \pm 27.7 \\
44.6 \% \\
n=10\end{array}$ \\
\hline $\begin{array}{c}\text { Invertzucker } \\
20 \% \mathrm{ig}\end{array}$ & $\begin{array}{l}49.2 \mathrm{mg} \pm 3.4 \\
18.9 \% \\
n=10\end{array}$ & $\begin{array}{l}54.8 \mathrm{mg} \pm 4.5 \\
21.1 \% \\
n=10\end{array}$ & $\begin{array}{l}85.1 \mathrm{mg} \pm 2.4 \\
32.8 \% \\
n=10\end{array}$ \\
\hline $30 \%$ ig & $\begin{array}{l}59.2 \mathrm{mg} \pm 4.3 \\
15.2 \% \\
n=10\end{array}$ & $\begin{array}{l}71.0 \mathrm{mg} \pm 5.3 \\
18.2 \% \\
n=10\end{array}$ & $\begin{array}{l}117.7 \mathrm{mg} \pm 10.2 \\
30.8 \% \\
n=10\end{array}$ \\
\hline $50 \%$ ig & $\begin{array}{l}83.0 \mathrm{mg} \pm 3.8 \\
12.8 \% \\
n=10\end{array}$ & $\begin{array}{l}100.7 \mathrm{mg} \pm 6.2 \\
16.5 \% \\
n=10\end{array}$ & $\begin{array}{l}157.7 \mathrm{mg} \pm 12.1 \\
24.2 \% \\
n=10\end{array}$ \\
\hline $\begin{array}{l}\text { Sorbit } \\
\qquad 3 \% \text { ig }\end{array}$ & $\begin{array}{l}23.8 \mathrm{mg} \pm 1.8 \\
61.2 \% \\
n=6\end{array}$ & $\begin{array}{l}26.5 \mathrm{mg} \pm 1.8 \\
68.0 \% \\
n=8\end{array}$ & $\begin{array}{l}34.2 \mathrm{mg} \pm 1.1 \\
87.4 \% \\
n=15\end{array}$ \\
\hline $\begin{array}{l}\text { Sorbit } \\
\qquad 5 \% \text { ig }\end{array}$ & $\begin{array}{l}36.0 \mathrm{mg} \pm 3.6 \\
55.4 \% \\
n=8\end{array}$ & $\begin{array}{l}37.8 \mathrm{mg} \pm 2.9 \\
58.1 \% \\
n=11\end{array}$ & $\begin{array}{l}51.7 \mathrm{mg} \pm 2.4 \\
79.1 \% \\
n=13\end{array}$ \\
\hline $10 \%$ ig & $\begin{array}{l}64.0 \mathrm{mg} \pm 9.4 \\
49.2 \% \\
n=9\end{array}$ & $\begin{array}{l}70.2 \mathrm{mg} \pm 6.3 \\
54.0 \% \\
n=13\end{array}$ & $\begin{array}{l}99.7 \mathrm{mg} \pm 4.8 \\
76.7 \% \\
n=14\end{array}$ \\
\hline $15 \%$ ig & $\begin{array}{l}91.1 \mathrm{mg} \pm 7.6 \\
46.8 \% \\
n=12\end{array}$ & $\begin{array}{l}96.7 \mathrm{mg} \pm 6.6 \\
49.6 \% \\
n=14\end{array}$ & $\begin{array}{l}118.2 \mathrm{mg} \pm 4.8 \\
61.0 \% \\
n=16\end{array}$ \\
\hline $\begin{array}{l}\text { Xylit } \\
\quad 3 \% \text { ig }\end{array}$ & $\begin{array}{l}21.1 \mathrm{mg} \pm 2.7 \\
54.4 \% \\
n=7\end{array}$ & $\begin{array}{l}26.1 \mathrm{mg} \pm 2.8 \\
66.9 \% \\
n=7\end{array}$ & $\begin{array}{l}30.7 \mathrm{mg} \pm 1.8 \\
78.7 \% \\
n=7\end{array}$ \\
\hline $5 \%$ ig & $\begin{array}{l}30.8 \mathrm{mg} \pm 3.0 \\
47.4 \% \\
n=10\end{array}$ & $\begin{array}{l}32.1 \mathrm{mg} \pm 4.2 \\
49.4 \% \\
n=8\end{array}$ & $\begin{array}{l}48.2 \mathrm{mg} \pm 2.9 \\
74.2 \% \\
n=7\end{array}$ \\
\hline $10 \%$ ig & $\begin{array}{l}50.8 \mathrm{mg} \pm 5.9 \\
39.1 \% \\
n=10\end{array}$ & $\begin{array}{l}68.9 \mathrm{mg} \pm 5.6 \\
53.0 \% \\
n=10\end{array}$ & $\begin{array}{l}85.8 \mathrm{mg} \pm 5.8 \\
66.0 \% \\
n=10\end{array}$ \\
\hline $15 \%$ ig & $\begin{array}{l}61.6 \mathrm{mg} \pm 7.4 \\
31.6 \% \\
n=8\end{array}$ & $\begin{array}{l}97.0 \mathrm{mg} \pm 13.2 \\
49.8 \% \\
n=9\end{array}$ & $\begin{array}{l}96.5 \mathrm{mg} \pm 5.0 \\
49.5 \% \\
n=8\end{array}$ \\
\hline
\end{tabular}

Die Differenz zwischen verabreichter Gesamtmenge und der im Magen wiedergefundenen Substanzmenge wurde als ,Darmangebot" bezeichnet und ist damit jene Größe, die Auskunft über das Ausmaß der Magenentleerung gibt. Der Übersichtlichkeit halber werden nur die Konzentrationen der jeweiligen Lösungen, das Darmangebot in mg und in \% der vorabreichten Gesamtmenge sowie die Zahl der Versuchstiere für die einzelnen Prüfzeiten angegeben.

diese Differenz aber insignifikant $(p>0.05)$. Dagegen ist die Differenz zwischen den Ergebnissen der beiden Hauptversuche statistisch signifikant $(p<0.01)$. Dadurch ist nachgewiesen, daß nicht nur der Glucosezu- satz allein, sondern auch die Menge der zum Kontrastmittel zugesetzten Glucose einen Einfluß auf die Magenentleerung ausübt (vergleiche Tabelle 2 mit Tabelle 3 ). 
Tabelle 2. Magenfüllung von 9 Probanden 2 Std nach Verabreichung von $250 \mathrm{ccm}$ Bariumsulfatlösung mit und ohne gleichzeitige Verabreichung von $50 \mathrm{~g}$ Glucose per os. (Die Kontrastmittelfläche auf den Röntgenbildern wurde planimetrisch gemessen)

\begin{tabular}{ccc}
\hline $\begin{array}{c}\text { Laufende } \\
\text { Nr. }\end{array}$ & $\begin{array}{c}\text { Vorversuch } \\
\text { ohne Glucose- } \\
\text { verabreichung }\end{array}$ & $\begin{array}{c}\text { Hauptversuch } \\
\text { mit Verabreichung } \\
\text { von } 50 \mathrm{~g} \text { Glucose }\end{array}$ \\
\hline 1 & $27.5 \mathrm{~cm}^{2}$ & $60.0 \mathrm{~cm}^{2}$ \\
2 & $25.0 \mathrm{~cm}^{2}$ & $65.0 \mathrm{~cm}^{2}$ \\
3 & $52.5 \mathrm{~cm}^{2}$ & $45.5 \mathrm{~cm}^{2}$ \\
4 & $24.0 \mathrm{~cm}^{2}$ & $44.0 \mathrm{~cm}^{2}$ \\
5 & $4.0 \mathrm{~cm}^{2}$ & $16.0 \mathrm{~cm}^{2}$ \\
6 & $2.5 \mathrm{~cm}^{2}$ & $37.5 \mathrm{~cm}^{2}$ \\
7 & $67.5 \mathrm{~cm}^{2}$ & $78.8 \mathrm{~cm}^{2}$ \\
8 & $2.0 \mathrm{~cm}^{2}$ & $2.0 \mathrm{~cm}^{2}$ \\
9 & $25.5 \mathrm{~cm}^{2}$ & $60.5 \mathrm{~cm}^{2}$ \\
Durchschnitts- & $25.61 \mathrm{~cm}^{2} \pm 7.48$ & $45.48 \mathrm{~cm}^{2} \pm 8.12$ \\
Wert: & & \\
Differenz innerhalb beider Versuchsreihen: $p<0.01$. \\
\hline
\end{tabular}

Tabelle 3. Magenfüllung von 10 Probanden 2 Std nach Verabreichung von $250 \mathrm{mg}$ Bariumsulfatlösung mit und ohne gleichzeitige Verabreichung von $100 \mathrm{~g}$ Glucose per os. (Die Kontrastmittelfläche wurde auf den Röntgenbildern planimetrisch gemessen)

\begin{tabular}{ccc}
\hline $\begin{array}{c}\text { Laufende } \\
\mathrm{Nr} .\end{array}$ & $\begin{array}{c}\text { Vorversuch } \\
\text { ohne Glucose- } \\
\text { verabreichung }\end{array}$ & $\begin{array}{c}\text { Hauptversuch } \\
\text { mit Verabreichung } \\
\text { von 100 g Glucose }\end{array}$ \\
\hline 1 & $4.0 \mathrm{~cm}^{2}$ & $100.0 \mathrm{~cm}^{2}$ \\
2 & $57.0 \mathrm{~cm}^{2}$ & $120.0 \mathrm{~cm}^{2}$ \\
3 & $27.3 \mathrm{~cm}^{2}$ & $112.5 \mathrm{~cm}^{2}$ \\
4 & $12.5 \mathrm{~cm}^{2}$ & $90.0 \mathrm{~cm}^{2}$ \\
5 & $2.0 \mathrm{~cm}^{2}$ & $75.0 \mathrm{~cm}^{2}$ \\
6 & $24.0 \mathrm{~cm}^{2}$ & $21.3 \mathrm{~cm}^{2}$ \\
7 & $12.0 \mathrm{~cm}^{2}$ & $52.5 \mathrm{~cm}^{2}$ \\
8 & $6.0 \mathrm{~cm}^{2}$ & $28.0 \mathrm{~cm}^{2}$ \\
9 & $3.0 \mathrm{~cm}^{2}$ & $56.0 \mathrm{~cm}^{2}$ \\
10 & $2.0 \mathrm{~cm}^{2}$ & $70.0 \mathrm{~cm}^{2}$
\end{tabular}

Durchschnitts-

wert: $\quad 14.98 \mathrm{~cm}^{2} \pm 5.47 \quad 71.53 \mathrm{c} \mathrm{m}^{2} \pm 10.62$ Differenz innerhalb beider Versuchsreihe: $p<0.001$

\section{Diskussion}

Verschiedene Autoren hatten sich mit der Beeinflussung der Magenentleerung durch den osmotischen Druck von per os verabreichten Lösungen beschäftigt. Bereits Ende des 19. Jahrhunderts konnte von MaRBAIX [20] wahrscheinlich gemacht werden, daß im Duodenum und im Jejunum Rezeptorzellen liegen, die bei hohem osmotischem Druck in diesen Darmabschnitten eine Hemmung der Magenentleerung bewirken. Die Regulation der Magenentleerung wird nach neueren Untersuchungen sowohl nervös als auch hormonal gesteuert [26, 27, 28]. Von RAVDIN et al. [29] war bereits früher bei Untersuchungen an Hunden nachgewiesen worden, daß Lösungen, die in den Darm gelangen, leicht hypoosmolar gegenüber dem Blut sind. Es war dabei gleichgültig, welche Konzentration die in den Magen eingebrachten Lösungen besaßen. REYNELL u. SpRAY [30] fanden bei normalen Ratten nach Applikation von Glucoselösungen per Schlundsonde keine Glucoselösungen per Schlundsonde keine Glucose in den unteren Darmabschnitten. Diese Ergebnisse konnten von uns in früheren Arbeiten bestätigt werden [21, 22, 23, 24]. Zusammen mit den anderen Untersuchungen ist erkennbar, daß die Resorptionsprüfung mittels der Schlundsondenmethode, wie sie z.B. von CoRI [3] empfohlen wurde, keine eigentliche Resorptionsprüfung darstellt, sondern in erster Linie eine Aussage über das Ausmaß der Magenentleerung erlaubt.

Die Magenentleerung kann auch medikamentös beeinflußt werden. Eigene Untersuchungen ergaben, daß z.B. Butylbiguanid eine gewisse Magenentleerungshemmung bewirken kann [9]. Daneben führten Ganglienblocker sowie Reserpin und Dihydroergotamin, parenteral an Ratten verabreicht, zu starken Einschränkungen dieser Funktion [6]. Auch Anoxämie kann die Magenentleerung hemmen [4]. Dagegen bewirken sowohl hypoglykämische Zustände $[10,19,23]$ wie auch - unter bestimmten Bedingungen - ein Diabetes mellitus eine Steigerung der Magenentleerung $[5,10]$.

Fwnton [7] stellte im Gegensatz zu CoRI [3] eine Abhängigkeit der intestinalen Resorption von der Konzentration der verabreichten Lösungen bei Ratten fest. Bei Applikation von Glucoselösungen in steigenden Konzentrationen nahm die Menge, die aus dem Magen entleert wurde, ab. Damit bestätigte er Untersuchungsergebnisse von Quigliy u. PhelPs [26], wonach konzentrierte Glucoselösungen in Kontakt mit dem Duodenum die Motilität des Magens hemmen. Die Magenentleerung war etwa der Quadratwurzel der Konzentration der verabreichten Lösung umgekehrt proportional. Diese Ergebnisse konnten, wie oben ausgeführt, auch von uns bestätigt werden.

In größeren Versuchsreihen hatte SHaY [31] mittels serienmäßiger Röntgenaufnahmen Untersuchungen am Menschen durchgeführt. Durch seine Versuchsanordnung ließen sich die früheren Vermutungen von MaRBAIX [20] bestätigen. Mittels einer Duodenalsonde brachte SHAY die Prüflösungen direkt in das Duodenum und konnte ihre Wirkung auf die Magenentleerung von Bariumbrei an der Röntgenkontrastaufnahme direkt messen. Es ergab sich, daß hypertonische Lösungen im Duodenum (5\% Kochsalz oder $40 \%$ Glucose) die Magenentleerung völlig aufhoben. $5 \%$ ige Glucoselösungen oder $0.9 \%$ ige Kochsalzlösungen hatten dagegen keinerlei Wirkung. Bei dieser Versuchsanordnung bewirkte auch das Einbringen von Wasser in das Duodenum eine Hemmung der Entleerung des Magens.

Hunt $[13,14,15]$ befaßte sich in mehreren Arbeiten mit der Steuerung der Magenentleerung. Dieser Autor spülte den Mageninhalt am Ende der Versuchsperiode mit einer eingestellten Phenolrotlösung aus. An der Konzentrationsänderung des Phenolrots ließ sich die im Magen vorhandene Flüssigkeitsmenge messen. Auch HuNT stellte fest, daß sich der Magen um so langsamer entleert, desto höher der osmotische Druck des Mageninhalts ist. Bei einer Mahlzeit mit hohem osmotischem Druck (es wurden bei diesem Versuch zwei flüssige Mahlzeiten gegeben, die erste enthielt $35 \mathrm{~g}$ Saccharose pro Liter, die zweite $200 \mathrm{~g}$ Saccharose pro Liter) war der Prozentsatz der den Magen verlassenden Menge 
pro Minute kleiner als bei derjenigen mit niedrigem osmotischem Druck. Auch bei Verabreichung von Glucose, Sorbit, Calciumchlorid, Ammoniumsulfat und Kaliumchlorid in wäßriger Lösung stellte HunT fest, daß das Volumen des nach 20 min noch vorhandenen Mageninhalts größer, d.h. die Magenentleerung verzögert war, wenn die Konzentration der gelösten Teilchen erhöht wurde. Es bestehen nach HuNT keine Gründe für die Annahme, daß es im Duodenum ebenfalls Rezeptoren gibt, die die Magenentleerung beschleunigen. Für die Hemmung der Magensekretion dürfte es besondere Rezeptoren geben, die auf Säuren und Zucker reagieren. Der Gebrauch des Ausdrucks „Osmorezeptor" wird damit gerechtfertigt, daß der Rezeptor mit Dextrose, Sorbit, Calciumchlorid, Kaliumchlorid und Ammoniumsulfat, d.h. sowohl mit Nichtelektrolyten als auch mit Elektrolytlösungen proportional zum osmotischen Druck der zugeführten Lösungen zu reagieren scheint. Eine Beschleunigung der Magenentleerung durch hypertonische Natriumchloridlösungen (1.1-1.5 $n \mathrm{NaCl}$ ) stellten Kubistova u. PoLACEK [17] fest. Weitere Versuche von Polacek zeigten, daß die Ursache hierfür die Konzentration von Natriumionen ist und keine Abhängigkeit vom osmotischen Druck bestehen soll.

Lawson u. Dragstedt [18] konnten an Hunden nachweisen, daß durch Pylorektomie oder durch Denervierung des Pylorusbereichs die Magenentleerung nicht verändert wurde. Wurde dagegen der gesamte Magen denerviert, so kam es zu einer starken Hemmung der Magenentleerung. Nur bei diesen Tieren führte Pylorektomie zu einer geringen Verbesserung der Magenentleerung. Der Pylorus scheint also nach diesen Untersuchungen nur eine geringe Rolle zu spielen. Die Magenentleerung würde hauptsächlich durch die Motorik des Magens bewirkt. Allerdings haben diese Autoren Testmahlzeiten gegeben und nicht Lösungen verschiedener Osmolarität.

Bei der Auswertung der eigenen tierexperimentellen Befunde ließ sich eine gewisse Abhängigkeit der Magenentleerung von der Schnelligkeit der Resorption feststellen. Dies galt jedenfalls für die rasch resorbierbare Glucose, die nach $60 \mathrm{~min}$ bei weitem am schnellsten aus dem Magen in den Darm entleert wurde (siehe Abb. 1). Auch Galaktose und Fructose verhalten sich ähnlich; allerdings ist in dieser Position auch der langsam resorbierbare Sorbit zu finden. Man würde es sich also zu leicht machen, wenn man die Resorptionsgeschwindigkeit der einzelnen. Substanzen als alleiniges Kriterium einer beschleunigten oder verzögerten Magenentleerung ansehen würde. Hiergegen spricht neben dem Verhalten des Sorbit auch die Tatsache, daß die Magenentleerung der Galaktose gegenüber der etwa gleich schnell resorbierbaren und isoosmotischen Glucose verzögert ist.

Die anfängliche Magenentleerung direkt nach Einbringung von Lösungen in den Magen scheint relativ schnell vor sich zu gehen (siehe Tabelle 1). Andererseits sprechen aber auch die Osmorezeptoren rasch an; denn hochprozentige Lösungen werden bereits nach 15 min nur stark verlangsamt in den Darm entleert. Aus Tabelle 1 ist sogar zu ersehen, daß die Entleerungshemmung bei Verabreichung höherprozentiger Lösun-

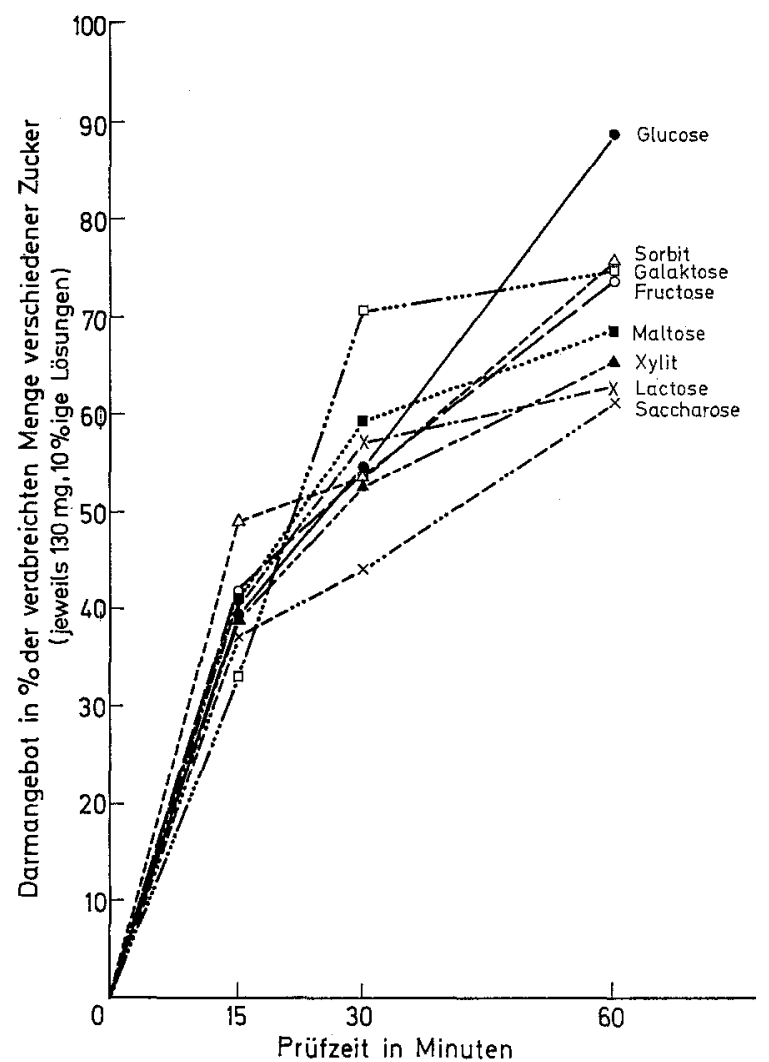

Abb. 1. Die Größe des Darmangebotes nach Verabreichung verschiedener Zucker per Schlundsonde an Ratten (Erklärung und Statisik siehe Text bzw. Tabelle 1)

gen bei der Prüfzeit „,15 min“ im Durchschnitt größer ist als bei der Prüfzeit , $60 \mathrm{~min}^{\prime c}$. Vergleicht man jeweils die Konzentrationen von $10 \%$ und von $50 \%$, so findet man folgende Werte für die Hemmung der Magenentleerung in Prozent bei 50\%igen gegenüber $10 \%$ igen Lösungen: Nach $15 \mathrm{~min}$ : Galaktose $29 \%$, Glucose $45 \%$, Fructose (30\%ige Lösung) $46 \%$, Saccharose $45 \%$, MaItose $62 \%$. Demgegenüber beträgt die Magenentleerungshemmung nach $60 \mathrm{~min}$. : Galaktose $36 \%$, Glucose $37 \%$, Fructose (30\% ige Lösung) $31 \%$, Sacharose $23 \%$, Maltose 36\%. Die Magenentleerungshemmung wird also mit Zunahme der Prüfzeit deutlich geringer. Dafür ist wahrscheinlich zu einem großen Teil die Verdünnungswirkung der Magensaftsekretion verantwortlich zu machen. Dieser Befund ist aber auch ein weiterer Hinweis dafür, daß der osmotische Druck der im Magen vorhandenen Lösungen die Magenentleerung bestimmt.

Folgendes weiteres Beispiel läßt sich für einen Einfluß des osmotischen Drucks auf die Magenentleerung anführen: Wenn man die Magenentleerung von Invertzucker und Saccharose, die ja beide chemisch Fructose und Glucose enthalten, vergleicht, dann ergibt sich, daß 
für den Invertzucker im Vergleich zu Saccharoselösungen gleicher Konzentration eine wesentlich langsamere Magenentleerung vorliegt (Abb. 2). Invertzucker, bei

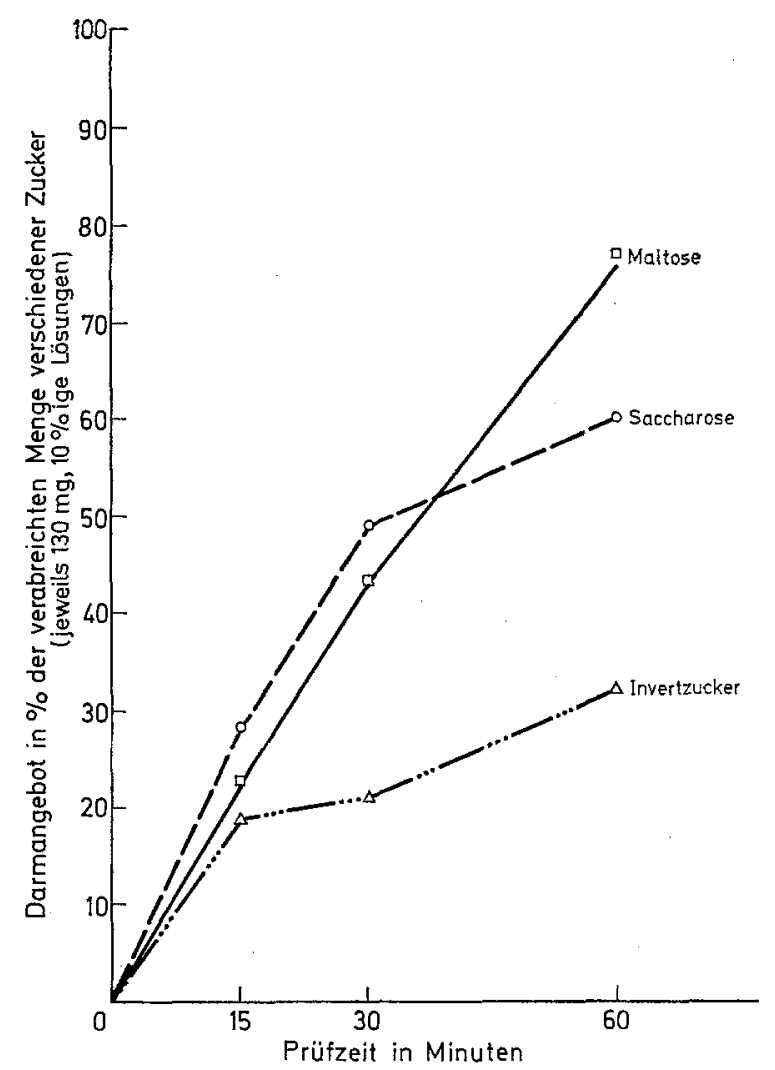

Abb. 2. Die Größe des Darmangebotes nach Verabreichung gleicher Mengen von Zuckern bei Lösungen verschiedenen osmotischen Drucks (Applikation per Sohlundsonde an Ratten). Erklärung und Statistik siehe Text bzw. Tabelle 1)

dem Glucose und Fructose in freier Form vorliegen, hat einen doppelt so hohen osmotischen Druck wie Saccharose.

Die Unterschiede in der Magenentleerung sind jedoch nicht allein - wenn auch zweifellos zu einem groBen Teil - durch den verschiedenen osmotischen Druck der eingebrachten Lösungen zu erklären. Nicht ohne weiteres verständlich ist z.B., warum die Disaccharide Maltose, Saccharose und Lactose bei gleichprozentigen Konzentrationen eine langsamere Magenentleerung aufweisen als Glucose, Galaktose und Sorbit, obwohl der osmotische Druck bei den Disaccharidlösungen gleicher Konzentration nur halb so groß ist. Wie schon oben bei der Abhängigkeit der Magenentleerung von der Resorptionsgeschwindigkeit diskutiert wurde, bestimmen individuelle Besonderheiten der einzelnen Prüfsubstanzen sicherlich auch das Ausmaß der Magenentleerung. In diesem Zusammenhang sei an die Ausführungen von Hunt erinnert (siehe oben), der ja ebenfalls für die von ihm postulierten Osmorezeptoren eine unterschiedliche Beeinflussung je nach Art der Prüfsubstanz fand.

Bei allen untersuchten Zuckern und Zuckeralkoho- len zeigte sich (siehe Tabelle 1), daß bei gleichen Konzentrationen der gleichen Substanz mit der Zeit eine Abnahme des Darmangebotes in \% erfolgt. Mit anderen Worten gibt also der Magen pro Zeiteinheit um so weniger Substanz an den Darm weiter, desto höhere Konzentrationen ihm angeboten werden. Dies ist als sinnvoller Regulationsmechanismus zu betrachten, der einer Überforderung der Resorptionskapazität und der daraus folgenden Diarrhoe entgegen wirken soll.

Von besonderer Bedeutung sind die Untersuchungen am Menschen. Auch wir konnten zeigen, daß der $\mathrm{Zu}$ satz von Glucose zu Bariumsulfatiösungen ein Sistieren der Magenentleerung im Vergleich zu den Vorversuchen bewirkt, bei denen kein Traubenzucker zusätzlich verabreicht wurde. Darüberhinaus ergeben sich Unterschiede hinsichtlich der Verabreichung von $50 \mathrm{~g}$ oder $100 \mathrm{~g}$ Glucose per os. Es ist zu erkennen, daß die Kontrastmittelfläche auf dem Röntgenbild bei Verabreichung von $100 \mathrm{~g}$ Glucose größer ist, als nach Verabreichung von $50 \mathrm{~g}$ Glucose. Die Durchschnittswerte besagen, dab in der ersten Probandengruppe, die $50 \mathrm{~g}$ Glucose erhielt, im Vorversuch die Kontrastmittelfläche im Durchschnitt $26 \mathrm{~cm}^{2}$ und nach $50 \mathrm{~g}$ Glucose $45 \mathrm{~cm}^{2}$ betrug. Während wir hier also eine Zunahme der Kontrastmittelretention um ca. $80 \%$ beobachteten, beträgt diese Zunahme bei Verabreichung von $100 \mathrm{~g}$ Glucose beinahe $400 \%$. Bei dieser zweiten Versuchsgruppe zeigte sich im Vorversuch eine durchschnittliche Kontrastmittelfläche von $15 \mathrm{~cm}^{2}$, nach $100 \mathrm{~g}$ Glucose von $72 \mathrm{~cm}^{2}$. Obwohl die Ergebnisse, wie aus den Einzelwerten ersichtlich ist, stark streuen, erlauben sie dennoch einen wichtigen Hinweis hinsichtlich der klinischen Diagnostik eines latenten Diabetes. Erstaunlicherweise werden nämlich für Glucosebelastungstests die gleichen diagnostischen Blutzuckergrenzwerte angegeben, gleichgültig, ob der Test mit $50 \mathrm{~g}$ oder $100 \mathrm{~g}$ Glucose per os durchgeführt wird [34]. Eine Erklärung hierfür liefert die bei unseren Untersuchungen festgestellte Verzögerung der Magenentleerung nach Verabreichung höherer Glucosemengen, die ein relativ geringes Angebot von Glucose pro Zeiteinheit an den Darm gewährleistet und damit die Blutglucosespiegel nicht so hoch ansteigen läßt, wie man es eigentlich erwartet. Aus unseren Untersuchungen kann nicht ersehen werden, ob es sich bei der Magenentleerung um eine Veränderung des Pylorustonus oder um eine veränderte Peristaltik im Antrum, wie es Lawson u. Dragstedt [18] annehmen, handelt.

Es läßt sich also feststellen, daß unter Berücksichtigung des Schrifttums und der eigenen, tierexperimentellen und der am Menschen gewonnenen Resultate dem osmotischen Druck eine bedeutsame Rolle für die Regulierung der Magenentleerung zukommt. Es muß offen bleiben, warum manche Substanzen bei einem Vergleich untereinander von dieser Gesetzmäßigkeit abweichen. Für sich allein betrachtet, folgt aber jede Substanz der Regel, daß bei höherer Konzentration der Lösungen eine Verzögerung der Magenentleerung zu beobachten ist. 


\section{Literatur}

1. Anneggers, J.H.: Net absorption of water, chloride and hexose from the intestine of dogs. Amer. J. Physiol. 200, 107-112 (1961).

2. ApperLy, F.L.: Duodenal regurgitation and the control of the pylorus. Brit. J. exp. Path. 7, 111-120 (1926).

3. CorI, C.F.: Fate of sugar in the animal body. 1. Rate of absorption of hexoses and pentoses from the intestinal tract. J. biol. Chem. 66, 691-715 (1925).

4. Crisler, G., and E.J. vax LIERE: The mechanism of the delay in gastric emptying time caused by anorexia. Amer. J. dig. Dis. 6, $221-224$ (1936).

5. Dotevalt, G.: Gastric emptying in diabetes mellitus. Acta med. scand. 170, 423-429 (1961).

6. DrzhevetskayA, I.A.: Alimentary hyperglycemia and intestinal glucose absorption following automatic ganglion block by ganglyolytic agents. Fed. Proc, Suppl. 22, 114-116 (1963).

7. Fenton, P.F.: Response of the gastrointestinal tract to ingested glucose solutions. Amer. J. Physiol. 144, $609-619(1945)$.

8. Förster, H., и. H. Mennert : Experimentelle Untersuchungen zur aktiven Resorption von Zuckern am lebenden Tier. I. Mitt. Zur Frage einer Substratsättigungskurve für die Resorption von Glucose und Galaktose. Klin. Wschr. 43, 834-839 (1965).

9. - E. Hager u. H. Mehnert : Der Einfluß von Butylbiguanid im Tierversuch auf die Resorption von Glucose und Fructose. Arzneimittel-Forsch. 15, 1340$1344(1965)$.

10. - , u. H. Mennert: Untersuchungen zur Beeinflussung der Glucose- und Fructoseresorption durch Insulin, Cortison und Thyroxin sowie Alloxandiabetes. Gastroenterologia 104 Suppl., 166 - 170 (1965).

11. Frank, H., u. E. KTRBERGER: Eine kolorimetrische Methode zur Bestimmung der ,,wahren Glucose" und Galaktose in $0.05 \mathrm{ml}$ Blut. Biochem. Z. 320, 359-368 (1950).

12. HuGgetr, A. St. G., and D.A. Nixon: Enzymic determination of blood glucose. Biochem. J. 66, $12 \mathrm{P}$. (1957).

13. Hunt, J.N., I. MaODonald, and W.R. SpurRet: The gastric response to pectin meals of high osmotic pressure. J. Physiol. 115, 185-195 (1951).

14. - Some properties of an alimentary osmoreceptor mechanism. J. Physiol. 132, 267-288 (1956).

15. - The osmotic control of gastric emptying. Gastroenterologya 41, 49-55 (1961).

16. - Die Steuerung der Magenentleerung. Triangel-Sandoz, Zschr. f. Med. Wissenschaft, 4, 266-271 (1960).

17. Kunistova, J., and E. Polacerk: The Evacuation of Glucose Solutions from the rat stomach during hypotonic hyperhydration. Acta Univ.-Carol. Med. Vol. 10, Nr. 3, 231-239, Praha 1964.
18. Lawson, L.J., and L.R. Dragstent: Pyloric effects on gastric secretion and emptying. Arch. Surg. 88, $1052-1058$ (1964).

19. Mackay, E.M., and W.C. Clark: The rate of glucose absorption from the intestine of the rat. Amer. J. Physiol. 135, 187-192 (1942).

20. Marbaix, O.: Le passage pylorique. Cellule 14, 249330 (1898).

21. Mehnert, H., u. H. Förster: Die Prüfung der Resorption von Glucose, Fructose und Sorbit mit einer neuen Methodik. Klin. Wschr. 39, 596-597 (1961).

22. - - Zur Prüfung der Resorption von Zuckern und Zuckeralkoholen bei Mensch und Tier. Gastroenterologia 104, Suppl., 101-106 (1965).

23. - - und G. VIGENER: Zur Frage der Wirkung von Insulin auf die Resorption im Dünndarm. Diabetologia (im Druck).

24. - - B. MeHnert, B. v. KutzschenbaCH, I. ThöRING u. K. ARBogast : Vergleichende Untersuchungen zur Resorption von Glucose, Galaktose und Lactose am Menschen und an der Ratte. I. Mitt.: Untersuchungsgut und Methodik, Voruntersuchungen und Kritik. Klin. Wsehr. 41, 540-543 (1963).

25. PolACEK, E.: On the question of osmotic influence on the stomach motility of rats. Acta univ. Carol. Med. 10, 65-74 (1964).

26. Qutgley, J.P., and K.R. Phesps: The mechanism of gastric motor inhibition from ingested carbohydrates. Amer. J. Physiol. 109, 133-140 (1934).

27. - , and H.S. Loukes: Effects of complete vagotomy on pyloric sphincter and the gastric evacuation mechanism. Gastroenterology 19, 533-538 (1951).

28. - - Gastric emptying. Amer. J. dig. Dis. 7, 672-676 (1962).

29. Radvin, I.S., C.G. Johrston, and P.J. Morrison: The absorption of glucose from the intestine. Amer. J. Physiol. 104, 700-709 (1933).

30. Reyneit, P.C., and G.H. SpRay: Absorption of glucose by the intact rat. J. Physiol. 134, 531-537 (1956).

31. Shay, H.: The pathology of gastric and duodenal ulcer. Bull. Acad. Med. N.Y. 20, 264-291 (1944).

32. Schubert, R., e. H. WAGNER: Normalausscheidung: von Periston $\mathrm{N}$ bei Anwendung eines neuen Kollidonnachweises. Z. ges. exp. Med. 131, 90-98 (1959).

33. WEST, C.D., and S. RAPOPORT: Modification of colorimetric method for determination of mannitol and sorbitol in plasma and urine. Proc. Soc. exp. Biol. N.Y. 70, $141-145(1949)$.

34. World Health Organization: Report of WHO Expert Comitee: Diabetes mellitus. Technical Report Series Nr. 310. Geneva 1965.

Priv.-Doz. Dr. H. Mehnert

III. Med. Abt. d. Krankenhauses

München Schwabing

8 München 23

Kölner Platz 1 